\title{
La eficiencia de los nuevos materiales fotosensibles usados en la fabricación de paneles solares
}

\section{The efficiency of the new photosensitive material used in the manufacture of solar panels}

\author{
Oriana Noguera-Salas \\ Ingeniera de Telecomunicaciones \\ Universidad Piloto de Colombia \\ Bogotá, Colombia \\ oriana-noguera@upc.edu.co
}

\author{
Ricardo Alfonso Pinto-García \\ M.Sc. en Informática Educativa \\ Universidad de La Sabana \\ Bogotá, Colombia \\ ricardo.pinto56@gmail.com
}

\author{
Jhon Edisson Villarreal-Padilla \\ M.Sc. en Ingeniería de la Información y las Comunicaciones \\ Universidad Piloto de Colombia \\ Bogotá, Colombia \\ jhon-villareal@unipiloto.edu.co
}

\begin{abstract}
Resumen- Las energías alternativas comienzan a tener preponderancia para ayudar en la mitigación del calentamiento global, y entre ellas la energía solar, por ser una fuente inagotable y de mayor masificación, dado a su buena relación beneficio-costo y facilidad de instalación y mantenimiento. Sin embargo, la eficiencia como medida de la energía eléctrica producida por una cantidad de radiación solar incidente, es baja. En este artículo se evalúan y analizan las características de los nuevos materiales fotosensibles que comienzan a utilizarse a fin de elevar la eficiencia de los paneles solares usados en hogares y en instalaciones de telecomunicaciones.
\end{abstract}

Los resultados que se obtienen llevan a establecer una comparación entre los materiales que actualmente se utilizan y los nuevos materiales que la industria comienza a emplear, donde se muestran aspectos que son relevantes, pero que no han sido evidenciados suficientemente.

Palabras Clave- Eficiencia, materiales fotosensibles, paneles solares, telecomunicaciones.

Abstract- The alternative energies begin to have prevalence to help in the mitigation of the global warming and between them the solar power for being an inexhaustible source, it is the one that more has put on attention for massif in view of his excellent results in cost and facility of installation and maintenance. Nevertheless, the results as for efficiency as measure of the electric power produced by a quantity of solar incidental radiation, are low. In this article, there are evaluated and analyze the basic characteristics of the new photosensitive materials that begin to be in use in order to raise the efficiency of the solar panels used in homes and in facilities of telecommunications.
The results that are obtained lead to establishing a comparison between the materials that nowadays are in use and the new materials that the industry begins to use, in order that from the point of view of the engineering they are considered to be some aspects that are relevant but that have not been demonstrated sufficiently.

Keywords- Efficiency, photosensitive materials, solar panels, telecommunications.

\section{INTRODUCCIÓN}

Las redes de telecomunicaciones en su infraestructura usan sistemas, equipos y elementos que deben ser alimentados con energía eléctrica para su operación, y estas fuentes de energía deben ser confiables y estables para que garanticen la operación y los servicios. Desde hace años se han venido usando sistemas de energía solar como fuentes alternativas complementarias que representan un avance en la consecución de estabilidad, de bajo costo y de impacto en la conservación del ambiente y mitigación del calentamiento global [1] [2].

La energía eléctrica suministrada por paneles solares se consigue mediante la conversión de la radiación solar que impacta celdas fotosensibles a energía eléctrica constante o corriente directa, que debe ser almacenada en baterías, para luego convertirla en energía alterna con los potenciales necesarios para alimentar los equipos de telecomunicaciones o los electrodomésticos en el hogar [3]. 
Las celdas solares hoy en día utilizan materiales semiconductores como el silicio y el grafeno, los cuales poseen características de buena conductividad y bajo consumo, lo que redunda en buena eficiencia. Sin embargo, el proceso de preparación de estos materiales es de muy alto costo y demanda muchos procesos para lograr un desempeño aceptable, porque en la práctica, los paneles fabricados con silicio no alcanzan una eficiencia mayor al 30\% [4]. Es así como en el mundo científico se han realizado investigaciones para encontrar nuevos materiales fotosensibles que posean unas mejores características que permitan incrementar ostensiblemente la eficiencia de los paneles a un bajo costo [5].

\section{LA RADIACIÓN SOLAR SOBRE LA TIERRA}

Existen dos tipos de radiación: la radiación directa, es decir, se da en línea recta y la radiación difusa, es decir, proviene de diferentes direcciones a causa de las nubes, turbidez atmosférica, la cual ayuda a aumentar la radiación irradiada en comparación con la radiación directa, y la suma de estas dos radiaciones crea la radiación global. Por ejemplo, en un día soleado, es más pronunciada la radiación directa siempre y cuando la elevación del sol sea superior a los $37^{\circ}$, de lo contrario será más pronunciada la radiación difusa.

Existen otros componentes de la radiación, la radiación infrarroja, la cual da gran parte de energía y la radiación ultravioleta relacionada principalmente al envejecimiento de los materiales [6].

Bajo la radiación directa, los cuerpos son capaces de proyectar una sombra, la cual se va moviendo conforme va cambiando el ángulo solar, lo que no ocurre con la radiación difusa.

Fig. 1. INCIDENCIA DE LA RADIACIÓN SOLAR POR ESTACIONES

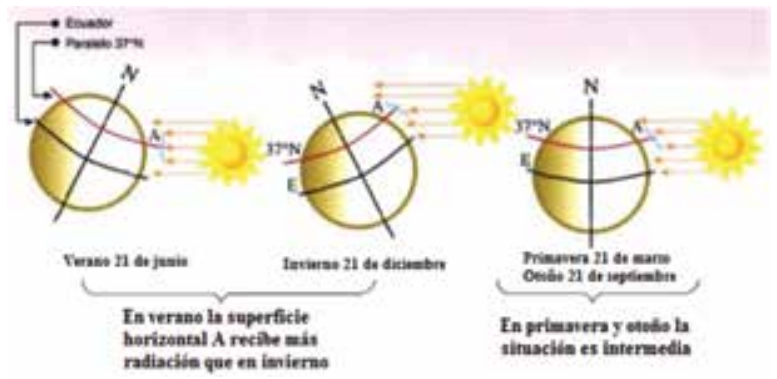

Fuente: http://www.magrama.gob.es/ministerio/pags/biblioteca/revistas/ pdf_Hort/Hort_2001_157_18_27.pdf [Citado el: 12 de junio de 2015].
Como se puede observar en la Fig. 1, la cantidad de energía solar que incide sobre la superficie terrestre depende principalmente del ángulo de inclinación de los rayos solares; mientras más perpendiculares sean, mayor será la intensidad, esta perpendicularidad depende de la posición geográfica de la región, es decir, la latitud, y de la época del año y la hora del día.

Fig. 2. ELEVACIÓN SOLAR

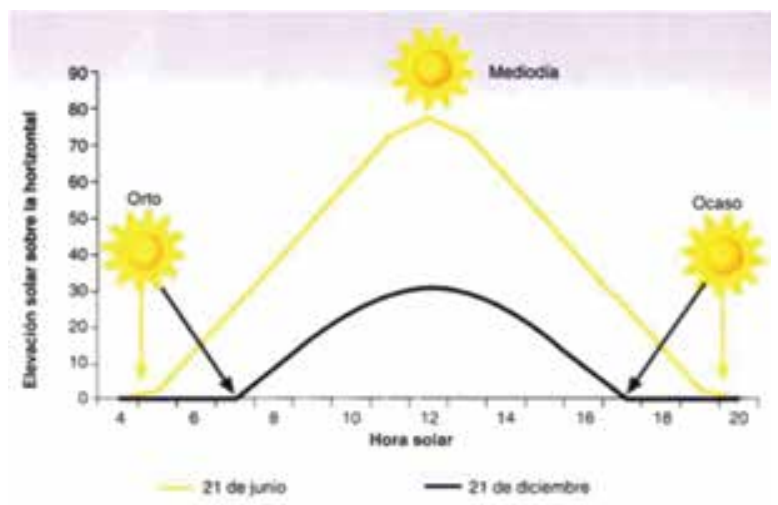

Fuente: http://www.magrama.gob.es/ministerio/pags/biblioteca/revistas/ pdf_Hort/Hort_2001_157_18_27.pdf [Citado el: 04 de agosto de 2016].

De igual forma en la Fig. 2, se ve que en fechas cercanas al 21 de diciembre, es decir, invierno, la duración del Sol es de 9 horas y la elevación es de máximo $30^{\circ}$ lo que indica una menor radiación solar sobre la superficie terrestre. Por otro lado, durante las fechas cercanas al 21 de junio, es decir, verano que la duración del Sol será de aproximadamente 15 horas y con una elevación de máximo $78^{\circ}$, lo que indica una mayor radiación solar sobre la superficie terrestre [6].

Existen dispositivos y sistemas que ayudan a evaluar la cantidad de radiación solar sobre las regiones terrestres, como son los satélites ambientales geoestacionarios que registran mediante imágenes tomadas cada cierto tiempo el estado de dicha energía sobre la Tierra. Para lograrlo se han elaborado modelos físicos (por ejemplo, especificación de parámetros basados en la transmisión de una función espectral en relación con la radiación existente en la atmósfera) que permiten entregar datos más exactos de las lecturas térmicas en esas zonas del mundo. Así, en Francia se realizó un estudio para utilizar el modelo estadístico-físico Heliosat II, el cual se basó en el uso de imágenes satelitales para medir la irradiancia global diaria y mensual. Este modelo lo financió la Unión Europea desde 1996 hasta 1998, con el 
objetivo de crear una base de datos que ayudara a mejorar, desarrollar y validar modelos de irradiación solar a nivel de superficie terrestre [7].

Ahora bien, en Colombia existe un patrón de radiación el cual ha sido previamente determinado por la UPME (Unidad de Planeación Minero-Energética).

Fig. 3. MAPA DE RADIACIÓN SOLAR GLOBAL ANUAL

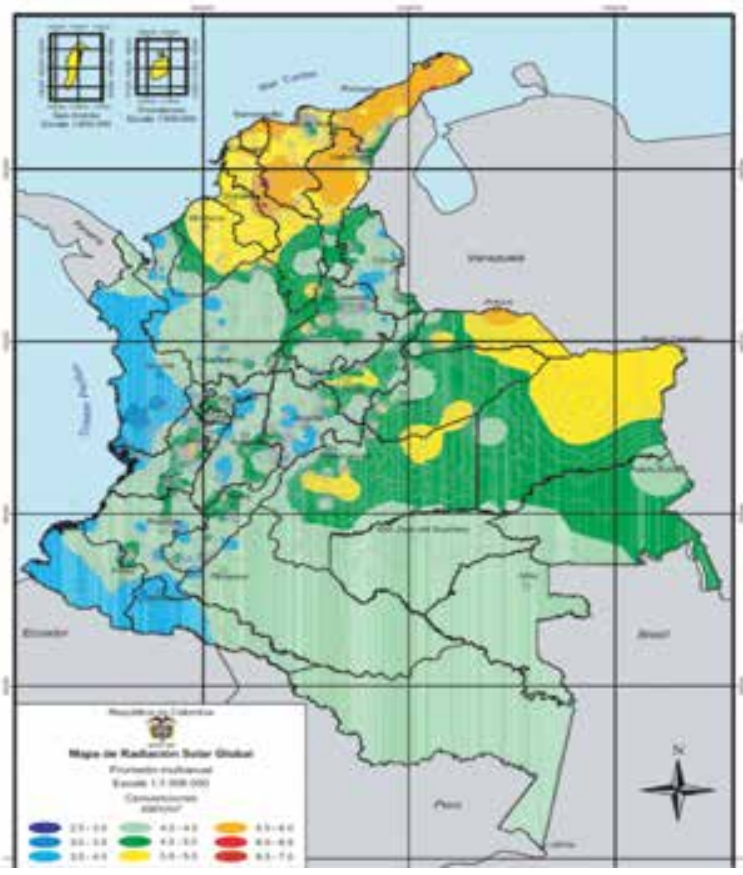

Fuente: UPME. 2010. Mapas de radiación solar global sobre una superficie plana. Bogotá: s.n., 2010. [Citado el: 09 de febrero de 2016].

En la Fig. 3 se observan las conversiones de colores relacionadas a los rangos de radiación solar en Colombia medidas en $\mathrm{kW} / \mathrm{m}^{2}$. Puede verse que los colores tienen un intervalo entre sí de 0.5, lo que indica que la zona central del país posee un nivel de radiación medio, de entre 4.5 a 5.0 kW/ $\mathrm{m} 2$, mientas que la zona norte, desde Montería hasta Riohacha posee un alto nivel de radiación entre 5.5 y $6.0 \mathrm{~kW} / \mathrm{m}^{2}$. No obstante, la mayor parte del territorio colombiano se encuentra en un nivel medio-bajo de radiación solar, el cual se encuentra entre 4.0 y $4.5 \mathrm{~kW} / \mathrm{m}^{2}$ [8].

La región con mayor radiación solar en Colombia es la península de La Guajira, siendo el mes de julio el mes de mayor promedio de radiación (superior a $650 \mathrm{cal} / \mathrm{cm}^{2}$ por día) y el mes de diciembre el de menor promedio de radiación (530 $\mathrm{cal} / \mathrm{cm}^{2}$ por día). En segundo lugar, la región Andina en el mes de febrero cuenta con niveles de radiación de $480 \mathrm{cal} / \mathrm{cm}^{2}$ por día, los cuales en el mes de junio tienen un promedio de radiación 420 $\mathrm{cal} / \mathrm{cm}^{2}$ por día. Las zonas con niveles más bajos de radiación son la costa Pacífica y el piedemonte llanero, con promedios menores a $300 \mathrm{cal} / \mathrm{cm}^{2}$ por día; durante el año es poco lo que varían, siendo marzo y abril los meses de mayor radiación, y noviembre y diciembre los de menor radiación.

\section{EL EFECTO FOTOVOLTAICO}

Conocido como la emisión de electrones por un metal al tener contacto con radiación solar. Este efecto fue descubierto en 1887 por H. Hertz, quien descubrió que si los electrones emitidos circulan por un circuito que mide la intensidad de corriente y se le añade una tensión considerable, la polaridad de este puede cambiar.

Según esto, la energía de los fotoelectrones es independiente a la intensidad de la luz, la energía máxima de los fotoelectrones $\left(\mathrm{K}_{\max }=\mathrm{eV}_{0}\right)$ depende de la frecuencia o longitud de onda de la radiación incidente, y el número de fotoelectrones liberados será proporcional a la intensidad de luz [3].

Fig. 4. EFECTO FOTOELÉCTRICO

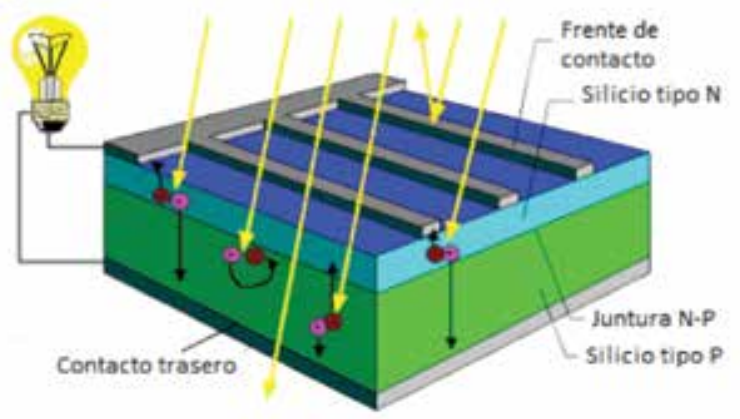

Fuente: http://cifes.gob.cl/otros/Generador\%20fotovoltaico.pdf. [Citado el: 17 de noviembre de 2015].

Como se observa en la Fig. 4, la radiación solar incide sobre las células fotovoltaicas agrupadas en módulos, los cuales la convierten en energía eléctrica. Los módulos son compuestos por celdas solares en las que la radiación solar incide sobre la unión PN del material semiconductor, causando un bombeo de fotones entre el frente de contacto hasta el contacto trasero.

Otro punto de vista de este fenómeno se puede dar mediante la siguiente explicación:

Los fotones tienen una energía característica determinada por la frecuencia de onda de la luz. 
Si un átomo absorbe energía de un fotón y tiene más energía que la necesaria para expulsar un electrón del material y al mismo tiempo posee una trayectoria dirigida hacia la superficie, el electrón puede ser expulsado del material. Mientras que si la energía del fotón es demasiado pequeña, el electrón no puede escapar de la superficie del material.

Los cambios de intensidad de luz no modifican la energía de sus fotones, sino que alteran el número de electrones que pueden escapar de la superficie sobre la que incide, y por tanto la energía de los electrones emitidos no depende de la intensidad de la radiación que llega, sino de la frecuencia [9].

\section{LAS CELDAS FOTOELÉCTRICAS}

Es el dispositivo del panel encargado de transformar la energía lumínica (radiación solar) en energía eléctrica por medio del efecto fotovoltaico.

Fig. 5. FUNCIONAMIENTO DE UNA CÉLULA FOTOVOLTAICA

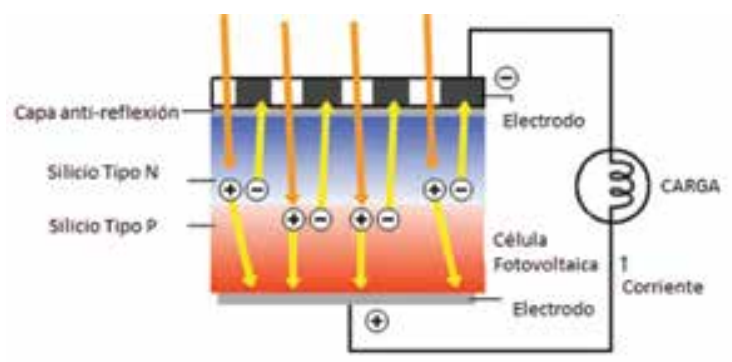

Fuente: http://oni.escuelas.edu.ar/2007/BUENOS_AIRES/1246/solar.htm. [Citado el: 23 de febrero de 2016].

Como se observa en la Fig. 5, los fotones provenientes de la radiación solar al impactar en la celda o célula, inciden sobre la primera capa (capa superficial) y son absorbidos por los materiales semiconductores (silicio, grafeno, etc.), lo cual genera un salto de electrones que causan un desplazamiento hacia la superficie de la célula y en el instante en que se desprenden los electrones se crean huecos o lagunas de carga positiva en la capa. En consecuencia se crean dos cargas, una positiva y otra negativa, las cuales generan una corriente eléctrica de manera constante en tanto se irradie luz solar sobre la superficie de la celda. Cada foto-celda provee aproximadamente 0.5 voltios de potencial.

Si se supone que sobre una celda con cierta área incide un espectro de radiación solar con $\mathrm{I}_{\mathrm{s}}(\mathrm{v})$ siendo la cantidad de fotones por unidad de área, cada segundo en cada banda unitaria de frecuencia, entonces la razón de pares electrón-hueco generados en el material al absorber los fotones de la radiación está dado por (1) [10]:

$$
G_{s}=\int_{v_{\min }} N(v) I_{s}(v) d v
$$

Donde:

$G_{s}$ es la razón de pares electrón-hueco

$N(v)$ es el número de pares electrón-hueco generados por cada fotón incidente

$V_{\min }=E_{g} / h$ donde, $h$ es la constante de Planck

Este factor $(N(v))$ involucra tanto la probabilidad de absorción de fotones como la generación adicional de pares electrón-hueco. Esta probabilidad es dependiente de la frecuencia $v$.

Cuando todos los portadores sean absorbidos por la unión $P N$ (eficiencia del 100\%), la densidad de corriente debida a la iluminación $\left(J_{L}\right)$ esta dado por (2)

$$
J_{L}=q \cdot G_{s}
$$

Donde:

$q$ es la magnitud de carga del electrón.

Existen varios tipos de celdas, por ejemplo, las celdas monocristalinas, las cuales son abundantes en la arena, donde cada célula es cortada con un fino espesor de silicio. Igualmente, están las celdas de tipo policristalino, las cuales cuentan con diferentes cortes de silicio. En comparación de estos tipos de celdas, las celdas monocristalinas son más eficientes en condiciones ideales de iluminación; sin embargo, es mejor en el momento en que el Sol está en un ángulo bajo sobre el panel. En teoría, el silicio monocristalino puede alcanzar un máximo del 32\%, y en estudios de laboratorio alcanzar un tope del $29 \%$, pero actualmente el silicio utilizado en módulos comerciales no sobrepasa el rango de eficiencia de 14 a 16\%. Mientras que el silicio policristalino no sobrepasa el rango de eficiencia de 13 a 15\%.

Actualmente existen paneles de 30, 33 o 36 celdas, los que superan este número necesitan un sistema de regulación, ya que alcanzan un voltaje excesivo [11]. 


\section{LA EFICIENCIA EN LAS CELDAS SOLARES}

Se le conoce como el porcentaje de potencia transformada en energía eléctrica de la luz solar. Este término puede calcularse relacionando la potencia máxima de la energía eléctrica generada $\left(P_{m}\right)$, con la irradiación de luz $\left(\mathrm{W} / \mathrm{m}^{2}\right)$ bajo ciertas condiciones específicas (STC) y el área superficial en $\mathrm{m}^{2}$ está dado por (3) [10].

$$
\eta=\frac{P_{m}}{E A_{c}}
$$

Donde:

$\mathrm{P}_{\mathrm{m}}$ es la potencia máxima.

E es la irradiación de luz.

$A_{c}$ es el área superficial.

Para definir el valor de eficiencia y cuánto puede llegar a variar, existen una serie de teorías: la teoría más completa es la teoría del balance detallado, la cual fue desarrollada por Shockley y Queisser [13], en la que establece que la corriente que produce una celda solar se determina por el número de fotones absorbidos y emitidos por el dispositivo (celda); esto es debido a que los fotones absorbidos determinan la fotocorriente, mientras que los emitidos relacionan el voltaje de operación. En la Fig. 6 se puede observar que los fotones de alta energía inciden en el espectro solar generando portadores, los cuales pasan por la banda de conducción con energía en exceso, la que ceden a otro par electrón-hueco.

Fig. 6. ESQUEMA BALANCE DETALLADO

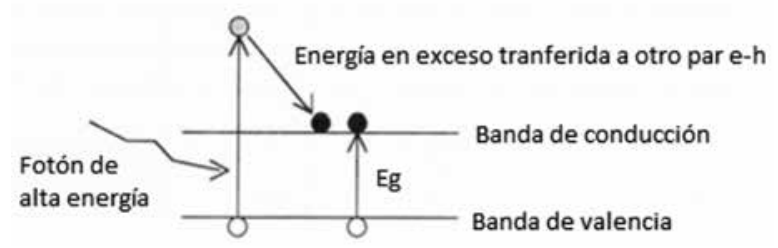

Fuente: Límites para la máxima eficiencia de celdas solares de silicio: efectos debidos a la generación Auger y a niveles profundos. Revista Mexicana de Física, 1996.

Gracias a esta teoría se ha podido calcular la eficiencia máxima para Silicio en el orden de $30 \%$ a $31 \%$ bajo radiación solar.

Esta teoría tiene un principio que la rige, el cual dice que la densidad de corriente que fluye por la celda será determinada por la razón de generación de pares electrón-hueco menos la recombinación total en desequilibrio, por lo cual si fr es la fracción de pares electrón-hueco, se define (4)

$$
\frac{J}{q}=G_{s}-\frac{R_{d}(V)-R_{d}(0)}{f_{r}}
$$

Donde,

$R_{d}(V)$ es la razón de recombinación radiativa en la celda

$\mathrm{R}_{\mathrm{d}}(0)$ es la radiación generada por la celda

Donde, si es un circuito abierto se tendrá a $\mathrm{J}=0$, según (5)

$$
R_{d}\left(V_{o c}\right)=f_{r} G_{s}+R_{d}(0)
$$

Por lo tanto, el voltaje del circuito abierto $\mathrm{V}_{\text {oc }}$ esta dado por (6)

$$
V_{o c}=V_{T} \operatorname{In}\left(\frac{f_{r} G_{s}}{R_{0 T}}+1\right)
$$

Por lo que se entiende que el voltaje máximo en el circuito abierto se obtendrá cuando $f_{r}=1$, es decir, cuando la recombinación de pares electrón-hueco sea nula y toda la recombinación sea causada por la emisión de radiación desde la celda a una temperatura T [10].

\section{LOS NUEVOS MATERIALES FOTOSENSIBLES}

En los últimos años se han venido realizando una serie de investigaciones para descubrir materiales que tengan propiedades fotovoltaicas y utilizarlos en la creación de nuevas celdas solares. Uno de esos materiales encontrados es el dióxido de titanio $\left(\mathrm{TiO}_{2}\right)$, el cual, por condiciones termodinámicas, su estructura no es apta para el oxígeno, permite una mejor absorción de luz solar y en consecuencia en la celda puede ceder una mayor cantidad de electrones al semiconductor, dando como resultado una mayor concentración de energía eléctrica [14].

La celda solar Graetzel es una celda solar basada en dióxido de titanio $\left(\mathrm{TiO}_{2}\right)$ o célula de pigmento fotosensible, creada por el inventor Michael Graetzel en 1991. El principio de funcionamiento de esta celda tuvo en cuenta el fenómeno de obtención de la clorofila que usan las plantas a partir de la intervención de la radiación solar. Tiene una estructura de dos electrodos planos: uno de vidrio, debido a su conductividad, y otro de nano-cristales de dióxido de titanio [15]. Usa el principio fotoelectroquímico de conversión de energía solar en energía eléctrica. 
Otro material que ha comenzado a tomar fuerza en la implementación de celdas solares es el Cobre $\mathrm{(Cu}$, caracterizado por su color rojizo con tonalidades brillantes y su capacidad de ser maleable, dúctil y un muy buen conductor. Normalmente es extraído en forma de sulfuro u óxidos. Es resistente a la atmósfera, agua limpia y agentes químicos externos. Una de las mayores ventajas del cobre es que es amagnético, es decir, no tiene magnetismo, lo cual permite que no sea atraído por los polos. Debido a que es capaz de transportar conductividad eléctrica y calor; tiene la capacidad de formar aleaciones metálicas, por lo que tiene una fuerte demanda en términos de construcción. A pesar de ser un elemento tan utilizado, posee un nivel de toxicidad a considerar, debido a que hay ciertos materiales que contienen sulfato de cobre, al inhalar o beber este material, puede ocasionar efectos como vómito, náuseas, diarrea, sudoración, entre otras cosas, sin embargo, no hay un elemento del cobre lo suficientemente tóxico como para generar la muerte en una persona, a menos que sea en porciones mayores a gramos [16].

Existe otro material llamado Perovskita [17]. Las perovskitas son materiales cristalinos formados por moléculas de elementos metálicos y no metálicos y formados por enlaces iónicos y/o covalentes. Son frágiles y con baja ductilidad. Pueden ser buenos aislantes eléctricos y térmicos debido a la ausencia de electrones conductores a temperatura ambiente. Poseen altas temperaturas de fusión y, así mismo, una estabilidad relativamente alta en la mayoría de los medios agresivos por causa de la estabilidad de sus enlaces fuertes [18].

Una de las propiedades físicas de las perovskitas son la ferroelectricidad y piezoelectricidad. En este tipo de perovskitas la aplicación de un campo eléctrico da como resultado la deformación del cristal y en forma recíproca, la deformación mecánica del cristal genera un campo eléctrico en el interior del material. Este fenómeno es conocido como piezoelectricidad y es una característica de todos los ferroeléctricos; sin embargo, no todos los piezoeléctricos presentan la propiedad de la ferroelectricidad [19].

\subsection{Cuadro informativo y comparativo de nuevos materiales fotosensibles}

En la actualidad se han realizado investigaciones relacionadas con el incremento de eficiencia en los paneles solares, de las cuales se descubrieron una serie de materiales fotosensibles como el dióxido de titanio $\left(\mathrm{TiO}_{2}\right)$, la perovskita, el cobre $(\mathrm{Cu})$, entre otros; cuyas características permiten un incremento en la recolección y transformación de energía.

Teniendo en cuenta las propiedades de estos materiales, se encontraron propiedades en común, por lo cual se hara un cuadro informativo en la Tabla I en el que se definirán dichas propiedades y un cuadro comparativo en la Tabla II identificando los valores de los materiales en cada propiedad.

TABLA I

DEFINICIÓN DE NUEVOS MATERIALES FOTOSENSIBLES

\begin{tabular}{|c|c|}
\hline \multicolumn{2}{|c|}{ PARÁMETROS DE EVALUACIÓN SOBRE LOS NUEVOS MATERIALES FOTOSENSIBLES } \\
\hline FLEXIBILIDAD & TOXICIDAD \\
\hline $\begin{array}{l}\text { Condición que posee algún material e inmaterial de moverse sin } \\
\text { rigidez, o de sufrir adaptaciones a los cambios o la movilidad, ser } \\
\text { maleable, etc. } \\
\qquad \frac{F}{S}=Y \frac{\Delta L}{L_{0}}\end{array}$ & $\begin{array}{l}\text { Es la capacidad que posee una sustancia o material químico de } \\
\text { perjudicar a un ser vivo. Indica la potencia de una sustancia ve- } \\
\text { nenosa, pero no su afección; puede expresarse en mg/kg de peso } \\
\text { vivo el cual origina determinados efectos en un tiempo dado. } \\
\text { Toxicidad aguda } \\
\qquad U T_{\alpha}=100 / C L_{50} \\
\text { Toxicidad crónica } \\
\qquad U T_{C}=100 / C E N 0\end{array}$ \\
\hline TEMPERATURA & CONDUCTIVIDAD \\
\hline $\begin{array}{l}\text { Magnitud física que refleja la cantidad de calor, ya sea de un cuer- } \\
\text { po, de un objeto o del ambiente. Dicha magnitud está vinculada a } \\
\text { la noción de frío (es decir, menor temperatura) y caliente (es decir, } \\
\text { mayor temperatura). } \\
{ }^{\circ} F={ }^{o} C * 1.8+32\end{array}$ & $\begin{array}{l}\text { Se conoce como la propiedad física presente en cuerpos, materiales } \\
\text { o elementos que hace que estos sean capaces de conducir electri- } \\
\text { cidad o calor. Es decir, permite tener la capacidad del paso libre de } \\
\text { electricidad sobre los materiales. }\end{array}$ \\
\hline
\end{tabular}

Fuente: Los autores 
TABLA II

CUADRO COMPARATIVO DE NUEVOS MATERIALES FOTOSENSIBLES

\begin{tabular}{|l|l|l|l|l|}
\hline \multicolumn{3}{|c}{ PARÁMETROS FíSICOS EN NUEVOS MATERIALES FOTOSENSIBLES } \\
\hline \multirow{2}{*}{ MATERIALES } & \multicolumn{4}{|c|}{ PARÁMETROS FísICOS } \\
\cline { 2 - 5 } & Flexibilidad & Toxicidad & Temperatura & Conductividad \\
\hline DIÓXIDO DE TITANIO & Medio & Bajo & Bajo & Bajo \\
\hline COBRE & Medio & Bajo & Alto & Muy alto \\
\hline PEROVSKITA & Medio & Bajo & Bajo & Alto \\
\hline
\end{tabular}

Fuente: Los autores.

Como se observa en la Tabla II, todos los materiales califican para la implementación de celdas solares, ya que todos cuentan con un nivel medio de flexibilidad y un nivel bajo de toxicidad, lo cual es muy importante a la hora de crear una celda solar, dado que es necesario que el material por utilizar cuente con la flexibilidad necesaria para moldearse según las características del panel. Gracias al nivel de toxicidad, la implementación de los materiales tendrá un riesgo mínimo en la salud y el desempeño del panel y el personal que lo manipule. Sin embargo, existen una serie de diferencias por resaltar entre estos materiales, las cuales son: 1) El cobre requiere trabajar en altas temperaturas mientras que el dióxido de titanio y la perovskita puedes ser moldeadas a bajas temperaturas, lo que facilita su manipulación y 2) El dióxido de carbono posee un bajo nivel de conductividad eléctrica, este es uno de los factores de mayor importancia debido a que se busca obtener un material que intensifique la conversión de luz solar en energía eléctrica para que de esta forma se amplifique la eficiencia de las celdas, mientras que la perovskita tiene un nivel alto de conductividad y el cobre un nivel muy alto de conductividad.

De lo anterior, se puede concluir que, el cobre es la mejor opción para la implementación de nuevas celdas fotosensibles debido a sus características, las cuales sobresalen de los demás materiales, seguidos de la perovskita que posee ventaja a nivel industrial por su fácil uso e implementación y bajo costo.

\section{SIMULACIÓN Y RESULTADOS}

Para poder determinar el nivel de eficiencia de los paneles solares, se realizó una simulación con determinados materiales fotosensibles con el fin de analizar cuál material genera más eficiencia a la hora de recolectar energía solar y transformarla en energía eléctrica.

En primera instancia, se tomó una serie de datos de consumo eléctrico real de una casa estrato medio-alto, como se observa a continuación en la Tabla III:

TABLA III

DATOS USO ELÉCTRICO PROMEDIO EN UNA VIVIENDA ESTRATO MEDIO-ALTO

\begin{tabular}{|c|c|c|c|c|c|c|}
\hline \multicolumn{7}{|c|}{ DATOS PANELES SOLARES } \\
\hline & ÍTEM & CANTIDAD & POTENCIA (W) & TOTAL POTENCIA (W) & CONSUMO (H) & ENERGÍA TEÓRICA (WH) \\
\hline 1 & Bombillo Led 10 W & 10 & 10 & 100 & 8 & 800 \\
\hline 2 & Televisor LCD 23" & 3 & 60 & 180 & 8 & 1440 \\
\hline 3 & Nevera Mediana & 1 & 600 & 600 & 24 & 14400 \\
\hline
\end{tabular}

Fuente: Los autores 


\subsection{Silicio monocristalino}

TABLA IV

CARACTERÍSTICAS FUNDAMENTALES DEL PANEL SOLAR

\begin{tabular}{|c|c|c|c|}
\hline \multicolumn{4}{|c|}{ CARACTERÍSTICAS DEL PANEL } \\
\hline \multicolumn{2}{|c|}{ Tipo } & \multicolumn{2}{|c|}{ Monocristalino } \\
\hline \multicolumn{2}{|c|}{$\begin{array}{l}\text { Mayor nivel de tolerancia } \\
\text { de salida }\end{array}$} & \multicolumn{2}{|c|}{$2,00 \%$} \\
\hline $\begin{array}{l}\text { Número de } \\
\text { celdas }\end{array}$ & 72 & $\begin{array}{l}\text { Número de } \\
\text { diodos bypass }\end{array}$ & 3 \\
\hline \multicolumn{2}{|c|}{ Coeficiente de voltaje } & \multicolumn{2}{|c|}{$-158,20 \mathrm{mV} / \mathrm{K}$} \\
\hline \multicolumn{2}{|c|}{ Coeficiente eléctrico } & \multicolumn{2}{|c|}{$5,30 \mathrm{~mA} / \mathrm{K}$} \\
\hline \multicolumn{2}{|c|}{ Coeficiente de emisión } & \multicolumn{2}{|c|}{$85,00 \%$} \\
\hline \multicolumn{2}{|c|}{ Coeficiente de absorción } & \multicolumn{2}{|c|}{$70,00 \%$} \\
\hline
\end{tabular}

Fuente: Los autores

Se hizo una simulación de 36.600 kW pico con un panel solar de tipo monocristalino como se puede ver en la Tabla IV. se calculó el nivel de potencia $(\mathrm{kW})$ según $(7)$

$$
\text { paneles } * P_{\max }=P_{\text {Total }}
$$

Donde,

$\mathrm{P}_{\max }$, es potencia nominal máxima.

Entonces se define (8)

120 paneles $* 305 \mathrm{~W}=36.600 \mathrm{Kwpico}$

Con este sistema se logró obtener el resultado mostrado en la Fig. 7, con una producción total anual de $39.437 \mathrm{~kW} / \mathrm{h}$.

Fig. 7. ENERGía ELÉCTRICA PRODUCIDA ANUALMENTE CON PANELES DE SILICIO MONOCRISTALINOS (39.437 kWh)

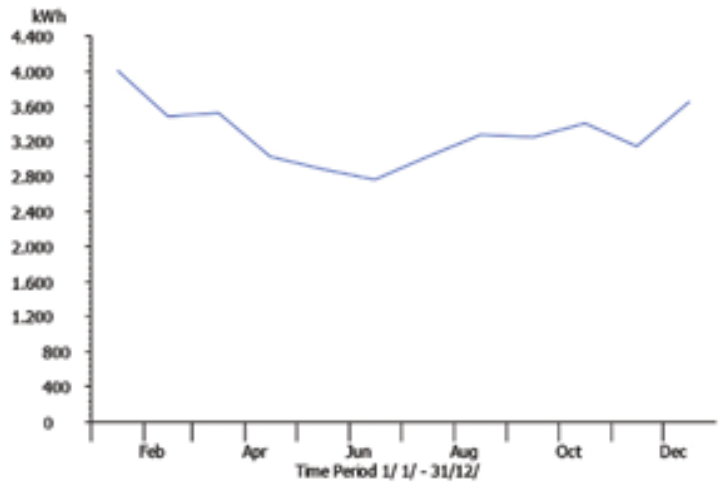

Fuente: Los autores.

\subsection{Silicio policristalino}

TABLA V

CARACTERÍSTICAS FUNDAMENTALES DEL PANEL SOLAR

\begin{tabular}{|c|c|c|c|}
\hline \multicolumn{4}{|c|}{ CARACTERÍSTICAS DEL PANEL } \\
\hline \multicolumn{2}{|c|}{ Tipo } & \multicolumn{2}{|c|}{ Policristalino } \\
\hline \multicolumn{2}{|c|}{$\begin{array}{l}\text { Mayor nivel de tolerancia } \\
\text { de salida }\end{array}$} & \multicolumn{2}{|c|}{$2,00 \%$} \\
\hline $\begin{array}{l}\text { Número de } \\
\text { celdas }\end{array}$ & 72 & $\begin{array}{l}\text { Número de } \\
\text { diodos bypass }\end{array}$ & 3 \\
\hline \multicolumn{2}{|c|}{ Coeficiente de voltaje } & \multicolumn{2}{|c|}{$-152,32 \mathrm{mV} / \mathrm{K}$} \\
\hline \multicolumn{2}{|c|}{ Coeficiente eléctrico } & \multicolumn{2}{|c|}{$5,83 \mathrm{~mA} / \mathrm{K}$} \\
\hline \multicolumn{2}{|c|}{ Coeficiente de emisión } & \multicolumn{2}{|c|}{$85,00 \%$} \\
\hline \multicolumn{2}{|c|}{ Coeficiente de absorción } & \multicolumn{2}{|c|}{$70,00 \%$} \\
\hline
\end{tabular}

Fuente: Los autores.

Se hizo una simulación de 36.600 kW pico con un panel solar de tipo policristalino como se puede ver en la Tabla V. Se calculó el nivel de potencia (kW) de acuerdo con (9):

$$
120 \text { paneles } * 350 W=36.600 K W \text { pico }
$$

Con este sistema se logró obtener el resultado mostrado en la Fig. 8, con una producción total anual de $40.072 \mathrm{~kW} / \mathrm{h}$.

Fig. 8. ENERGÍA ELÉCTRICA PRODUCIDA ANUALMENTE CON PANELES DE SILICIO POLICRISTALINOS (40.072 kWh)

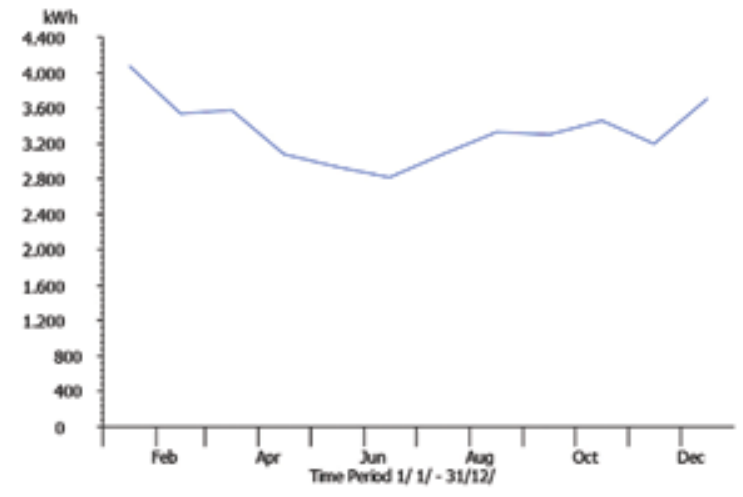

Fuente: Los autores. 


\subsection{Cobre}

TABLA VI

CARACTERÍSTICAS FUNDAMENTALES DEL PANEL SOLAR

\begin{tabular}{|c|c|c|c|}
\hline \multicolumn{4}{|c|}{ CARACTERÍSTICAS DEL PANEL } \\
\hline \multicolumn{2}{|c|}{ Tipo } & \multicolumn{2}{|c|}{ Cobre } \\
\hline \multicolumn{2}{|c|}{$\begin{array}{l}\text { Mayor nivel de tolerancia } \\
\text { de salida }\end{array}$} & \multicolumn{2}{|c|}{$2,00 \%$} \\
\hline $\begin{array}{l}\text { Número de } \\
\text { celdas }\end{array}$ & 120 & $\begin{array}{l}\text { Número de } \\
\text { diodos bypass }\end{array}$ & 3 \\
\hline \multicolumn{2}{|c|}{ Coeficiente de voltaje } & \multicolumn{2}{|c|}{$-111,29 \mathrm{mV} / \mathrm{K}$} \\
\hline \multicolumn{2}{|c|}{ Coeficiente eléctrico } & \multicolumn{2}{|c|}{$4,11 \mathrm{~mA} / \mathrm{K}$} \\
\hline \multicolumn{2}{|c|}{ Coeficiente de emisión } & \multicolumn{2}{|c|}{$85,00 \%$} \\
\hline \multicolumn{2}{|c|}{ Coeficiente de absorción } & \multicolumn{2}{|c|}{$70,00 \%$} \\
\hline
\end{tabular}

Fuente: Los autores.

Se realizó una simulación de 36.520 kW pico con un panel solar de tipo Cobre como se puede ver en la Tabla VI. Se calculó el nivel de potencia (kW) de acuerdo con (10)

$$
166 \text { paneles } * 220 W=36.520 K W \text { pico }
$$

Con este sistema se logró obtener el resultado mostrado en la Fig. 9, con una producción total anual de $46.883 \mathrm{~kW} / \mathrm{h}$.

Fig. 9. ENERGíA ELÉCTRICA PRODUCIDA ANUALMENTE CON PANELES DE COBRE (46.883 kWh)

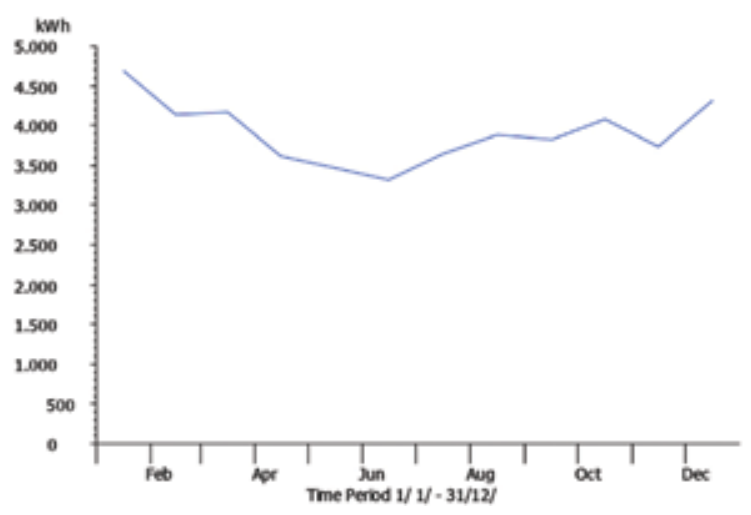

Fuente: Los autores.

\section{CONCLUSIONES}

Los nuevos materiales fotosensibles analizados y descritos en este artículo permitirán la fabricación de paneles solares con niveles de eficien- cia superiores a los que se pueden conseguir hoy en día comercialmente.

En el capítulo 7 se realizó un análisis de los paneles solares comerciales (paneles de silicio monocristalino, paneles de silicio policristalino y paneles de cobre) y se puede concluir que el panel tipo cobre genera $46,072 \mathrm{~kW} / \mathrm{h}$ al año con un sistema de $36.520 \mathrm{~kW}$ pico por lo cual el panel solar tipo cobre genera una mayor cantidad de energía eléctrica mediante la absorción de energía solar en comparación con un panel monocristalino o policristalino. Esto se logra incrementando el número de celdas. Después del panel tipo cobre, el tipo de panel de mayor eficiencia es el policristalino, donde con un sistema de $36,600 \mathrm{~kW}$ pico se genera $40.072 \mathrm{~kW}$ pico, comparado con el tipo de panel monocristalino que con un sistema de 36.600 kW pico genera 39,437 kW pico.

Para continuar con el proyecto de investigación se plantea que el siguiente paso es hacer un estudio financiero con el fin de analizar el costo beneficio de los diferentes tipos de paneles.

La implementación de este sistema de energía renovable tendrá principalmente impacto ambiental, ya que es una fuente de energía inagotable y de bajo impacto en contaminación, adicionalmente su funcionamiento no genera sustancias nocivas en el ecosistema, contribuye a la economía de un país porque impulsa el ahorro de energía eléctrica.

\section{REFERENCIAS}

[1] V. C. a. B. Sikdar, "Resource provisioning and dimensioning for solar powered cellular base stations," 2014 IEEE Global Communications Conference, pp. 24982503, 2014.

[2] E. C.-V. J. A. C.-N. a. M. R.-G. A. Rivera-Alfaro, "Sizing of an hybrid generation system as an primary energy source for a base transceiver station," 2014 IEEE Central America and Panama Convention (CONCAPAN XXXIV), Panama City, pp. 1-6, 2014.

[3] M. R. Meza, El Efecto Fotoeléctrico, [en línea]. Available: http://www.fisica.ru/dfmg/teacher/archivos/ efecto_fotoelectrico.pdf. [Último acceso: 09 Marzo 2016].

[4] T. G. P. a. A. Asokan, "A proficient solar panel efficiency measurement system: Using current measurements," International Conference on Communication and Electronics Systems (ICCES), pp. 1-6, 2016.

[5] J. Oviedo-Salazar, Historia y uso de energías renovables, México, 2015.

[6] J. Hernandez, "La radiación solar en invernaderos mediterraneos," Tecnología de producción, pp. 18-22, 2001. 
[7] J. Martínez P., Optimización de modelos de estimación de la radiación solar a partir de imágenes de satélite, Madrid, 2009.

[8] UPME, UPME: Mapas de radiación solar global sobre una superficie plana. [En línea]. 2010. [Último acceso: 09 Febrero 2016].

[9] Anónimo, Energía solar fotovoltaica. [En línea]. 2009. [Último acceso: 20 Septiembre 2016].

[10] A. Morales A., "Límites para la máxima eficiencia de celdas solares de silicio: efectos debidos a la generación Auger y a niveles profundos," Revista Mexicana de Física, pp. 449-458, 1996.

[11] E. I. Pereda, Celdas fotovoltaicas en generación distribuida, Chile, 2005.

[12] G. Ferro, E. Lentini y C. Romero, Eficiencia y su medición en prestadores de servicios de agua potable y alcantarillado. [En línea]. Febrero 2011. Available: http://www.cepal.org/publicaciones/xml/8/42728/ Icw385e.pdf.
[13] Anónimo, The Shockley-Queisser limit. [En línea]. 2008. Available: http://ph.qmul.ac.uk/sites/default/ files/u75/Solar\%20cells_environmental\%20impact. pdf. [Último acceso: 15 agosto 2016].

[14] T. G. Erika y A. Gutarra, Aplicaciones electrocrómicas y fotocatalíticas del diócido de titanio, 2004.

[15] Cited, Celdas de Grätzel, Medellín, 2012.

[16] G. Nordberg, "Materiales: Propiedades químicas y tóxicas," Productos químicos.

[17] W. Smith, Fundamentos de la Ciencia e Ingeniería de Materiales, Segunda ed., México: McGraw-Hill, 1993.

[18] A. J. Cuervo, Propiedades estructurales y espectroscopía de impedancia del estanato tipo perovskita," Bogotá, 2011.

[19] J. Roa-Rojas, "Propiedades y aplicaciones de nuevos materiales de tipo perovskita," Memorias de V Semana Ciencia y Tecnología. Universidad Francisco de Paula Santander, $n^{\circ}$ ISBN: 978-958-8489-001, p. 18, 2008. 